\title{
Instantaneous normal modes analysis of amorphous and supercooled silica
}

\author{
Scott D. Bembenek ${ }^{\mathrm{a})}$ \\ Department of Chemistry, Colorado State University, Fort Collins, Colorado 80523 \\ Brian B. Laird ${ }^{\text {b) }}$ \\ Department of Chemistry, University of Kansas, Lawrence, Kansas 66045
}

(Received 14 August 2000; accepted 8 November 2000)

\begin{abstract}
The dynamics of a model for amorphous and supercooled silica $\left(\mathrm{SiO}_{2}\right)$, a strong glass former, is studied using instantaneous normal mode (INM) analysis. The INM spectra at a variety of temperatures are calculated via molecular dynamics simulation. At temperatures below the glass transition temperature, the dominant contribution to the soft highly anharmonic modes comprising the imaginary frequency region of the INM spectrum are found to correspond to coupled rotations of $\mathrm{SiO}_{4}$ tetrahedral units, consistent with interpretations of neutron scattering experiments [B. B. Buchenau, H. M. Zhou, and N. Nucker, Phys. Rev. Lett. 60, 1318 (1988)] and with previous normal mode analysis of simulation results at $T=0 \mathrm{~K}$ [S. N. Taraskin and S. R. Elliot, Phys. Rev. B 56, 8623 (1997)]. (C) 2001 American Institute of Physics. [DOI: 10.1063/1.1337040]
\end{abstract}

\section{INTRODUCTION}

The dynamical behavior of glasses and amorphous materials, in general, is considerably richer than that of the corresponding crystals, leading to significant anomalous behavior in the thermodynamic and transport properties (e.g., specific heat or thermal conductivity) of these systems at low temperature. Currently, such effects are attributed to the presence of disorder-induced nonacoustic excitations that coexist with the acoustic phonons and that dominate the density of states at low energies. At very low energies (or low temperatures) the dominant modes are generally accepted to be localized two-level states (or tunneling modes). ${ }^{1-3}$ At higher temperatures, where anomalous behavior is still significant, but not explainable within the sample two-level system model, there is some evidence from experiment and simulation that the dominant excess modes are low-frequency (quasi)localized vibrations ${ }^{4-7}$ and that these modes and the tunneling modes share a common structural origin. ${ }^{8}$ However, a clear general microscopic picture is still lacking.

One of the most well studied of glasses is amorphous silica $\left(\mathrm{SiO}_{2}\right)$. In addition to its obvious technological importance, it is also of fundamental significance as the archetypical network solid and strong glass former. ${ }^{9}$ Neutron scattering experiments at low temperatures ${ }^{5}$ have observed low frequency vibrations in excess of the sound waves, which have been interpreted to be (quasi)localized coupled rotations of $\mathrm{SiO}_{4}$ tetrahedral units. This interpretation is consistent with recent computer simulation analysis by Taraskin and Elliot ${ }^{10}$ of the $T=0 \mathrm{~K}$ vibrational states for two model potentials for $\mathrm{SiO}_{2}$. In a later paper, ${ }^{11}$ they also report that the bare localized modes (obtained after subtracting out the acoustic phonon component in the quasilocalized eigenvec-

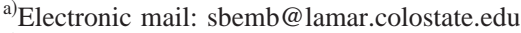

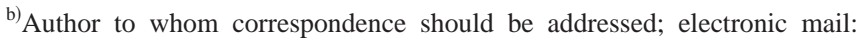
blaird@ukans.edu
}

tors) are strongly anharmonic even for small displacements.

The normal mode calculations of Taraskin and Elliot were necessarily restricted to analysis of quenched systems at zero temperature. Therefore, information on the existence, nature or importance of quasilocalized vibrational modes at higher temperatures, up to and beyond the glass transition temperature was not obtained. In this paper, we extend the analysis of Taraskin and Elliot to amorphous and supercooled silica at nonzero temperature by using instantaneous normal mode analysis, a technique that we have applied previously to simple monatomic, fragile glass formers, with some success. ${ }^{12,13}$

\section{INSTANTANEOUS NORMAL MODES}

Instantaneous normal modes (INMs) are a useful tool in analyzing condensed phases and have been used with varying degrees of success in studying the dynamics of normal liquids, ${ }^{14-20}$ as well as glasses and supercooled liquids. ${ }^{12,13,21,22}$ The INMs are defined in analogy with the more familiar normal modes. For an $N$-particle system at a given temperature $T$ one chooses a configuration (defined by a $3 \mathrm{~N}$-dimensional vector of atomic coordinates, $\mathbf{R}_{0}$ ) from the trajectory at some time $t_{0}$. Similar to standard normal mode analysis, the total potential is expanded in a Taylor series about $\mathbf{R}_{0}$ to yield

$$
\begin{aligned}
\Phi(\mathbf{R})= & \Phi\left(\mathbf{R}_{0}\right)-\mathbf{F} \cdot\left(\mathbf{R}-\mathbf{R}_{0}\right) \\
& +\frac{1}{2}\left(\mathbf{R}-\mathbf{R}_{0}\right) \cdot \mathbf{K} \cdot\left(\mathbf{R}-\mathbf{R}_{0}\right)+\cdots,
\end{aligned}
$$

where the $3 N$-dimensional force vector, $\mathbf{F}$ and the $3 N \times 3 N$ dynamical matrix, $\mathbf{K}$ are given by

$$
(\mathbf{F})_{i \alpha} \equiv-\left.\frac{1}{\sqrt{m_{i}}} \frac{\partial \Phi(\mathbf{R})}{\partial R_{i \alpha}}\right|_{\mathbf{R}=\mathbf{R}_{0}},
$$

and 


$$
\left.(\mathbf{K})_{i \alpha, j \beta} \equiv \frac{1}{\sqrt{m_{i} m_{j}}} \frac{\partial^{2} \Phi(\mathbf{R})}{\partial R_{i \alpha} \partial R_{j \beta}}\right|_{\mathbf{R}=\mathbf{R}_{0}},
$$

respectively. The atomic indices are labeled by $i$ and $j, \alpha$ and $\beta$ denote one of the Cartesian coordinates and $m$ is the mass of the atom. Since the configuration is chosen from the trajectory of a system at nonzero temperature, it will generally not be a (global or local) minimum on the many-body potential surface. Therefore, unlike the case of standard normal mode analysis, the force vector cannot be assumed to vanish and $\mathbf{K}$ is not necessarily positive definite. However, as in standard normal mode analysis, diagonalization of $\mathbf{K}$ to yield the instantaneous normal modes (eigenvectors) and the corresponding INM frequencies (the square roots of the INM eigenvalues) gives a description of the many-body potential energy surface and short-time dynamics based on independent motion along the INM eigenvectors. Since $\mathbf{K}$ can be nonpositive definite, the possibility exists to find negative eigenvalues, which result in imaginary frequencies as well as the usual positive eigenvalues (positive real frequencies). The imaginary frequency modes are highly anharmonic collective motions of the system. Although they cannot be directly interpreted on an individual basis as representing barrier crossing events, ${ }^{23}$ they do give a qualitative indication of the types of motions in the system that lead to structural relaxation, which is especially useful in the study of glasses and supercooled liquids. ${ }^{13}$

For a given temperature and density, the normalized INM density of states (DOS) is defined as

$$
\mathcal{D}(\omega) \equiv\left\langle\frac{1}{3 N} \sum_{i=1}^{3 N} \delta\left(\omega-\omega_{i}\right)\right\rangle,
$$

where the $\langle\ldots\rangle$ represent a configurational average. This configurationally averaged INM DOS can best be viewed as giving a statistically weighted representation of the curvature of the many-body potential surface.

In addition to the distribution of frequencies, it is often useful to consider the spatial distribution of a given INM. For example, in our earlier work on simple glasses, ${ }^{12,13}$ we found that the imaginary frequency modes of such glasses were localized in space for very low temperatures, with extended modes only appearing above the glass transition temperature. To quantify the spatial extent of the normalized eigenvectors ( $\mathbf{e}_{\alpha}^{i}$, of each INM, where $i$ runs over the $N$ particles in the sample and $\alpha$ labels the modes), we utilize a standard measure of localization, namely, the participation ratio, first introduced by Bell and Dean ${ }^{24}$

$$
p_{\alpha} \equiv\left[N \sum_{i=1}^{N}\left(\mathbf{e}_{\alpha}^{i} \cdot \mathbf{e}_{\alpha}^{i}\right)^{2}\right]^{-1} .
$$

For extended modes $p$ is of order unity while for localized or quasilocalized modes, it will scale inversely with the system size. To clarify, consider two limiting cases: if the mode is perfectly extended the total kinetic energy is equally shared among the atoms in the system resulting in $\left(\mathbf{e}_{\alpha}^{i} \cdot \mathbf{e}_{\alpha}^{i}\right)=(1 / N)$ and $p_{\alpha}=1$. However, if the mode is perfectly localized, where one atom has all the kinetic energy, then $\left(\mathbf{e}_{\alpha}^{i} \cdot \mathbf{e}_{\alpha}^{i}\right)$ $=\delta_{i 1}$ and $p_{\alpha}=(1 / N)$.

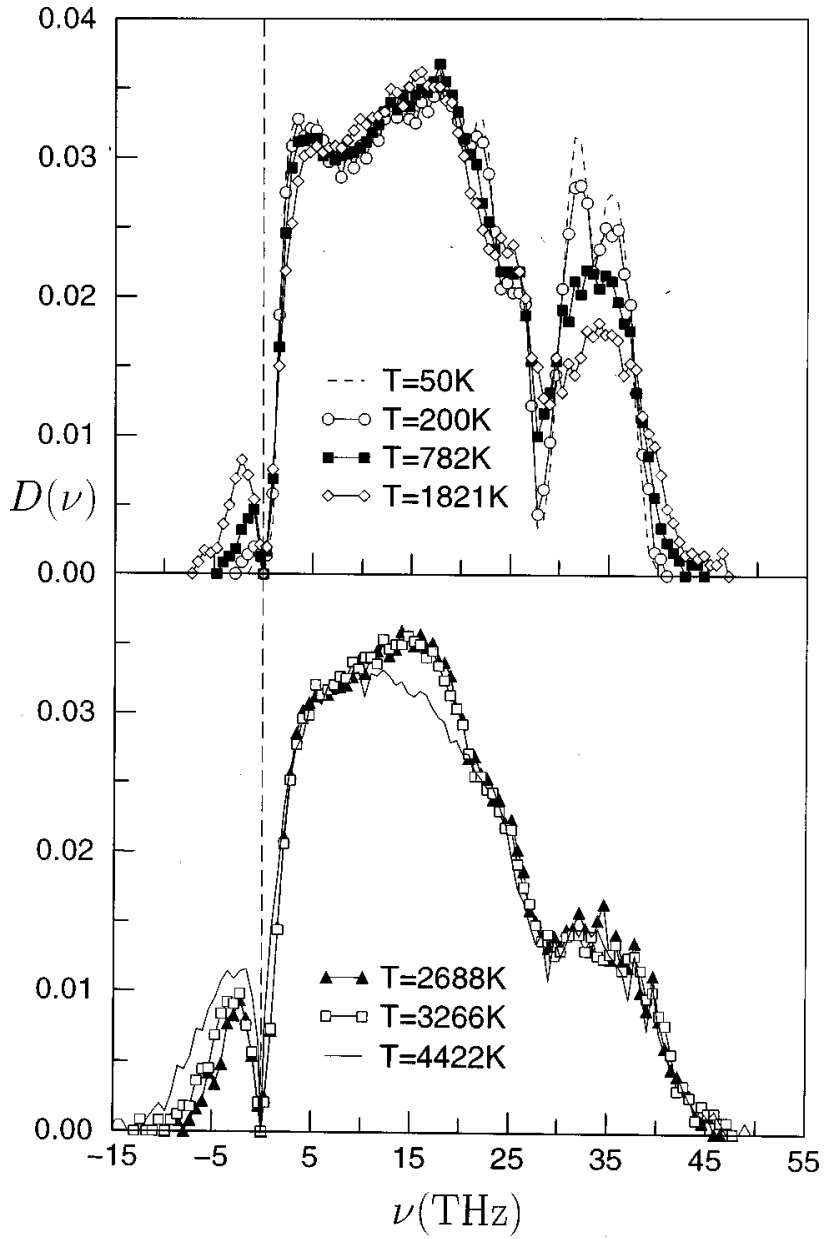

FIG. 1. The INM density of states as a function of frequency at various temperatures. For display purposes, the imaginary frequencies are plotted along the negative axis.

\section{RESULTS FOR A MODEL OF SILICA}

In our INM study of amorphous $\mathrm{SiO}_{2}$ (silica) we use the two-body potential developed by van Beest, Kramer, and van Santen (BKS), ${ }^{25}$ which has been shown to accurately describe silica in both its crystalline ${ }^{25}$ and amorphous ${ }^{26,10,27,11}$ forms. The form of the potential is given by a Coulomb term, a short-ranged interaction term defined by a repulsive exponential, and an attractive van der Waals tail. The potential between particle $i$ and $j$ is given by

$$
v\left(r_{i j}\right)=\frac{q_{i} q_{j} e^{2}}{r_{i j}}+A_{i j} e^{-b_{i j} r_{i j}}-\frac{c_{i j}}{r_{i j}^{6}},
$$

where $A_{i j}, B_{i j}$ and $C_{i j}$ are given by $A_{\mathrm{SiSi}}=0.0 \mathrm{eV}, A_{\mathrm{SiO}}$ $=18003.5773 \mathrm{eV}, A_{\mathrm{OO}}=1388.7730 \mathrm{eV}, B_{\mathrm{SiSi}}=0.0 \AA^{-1}$, $B_{\mathrm{SiO}}=4.87318 \AA^{-1}, B_{\mathrm{OO}}=2.76000 \AA^{-1}, C_{\mathrm{SiSi}}=0.0 \mathrm{eV} \AA^{5}{ }^{5}$ $C_{\mathrm{SiO}}=133.5381 \mathrm{eV} \AA{ }^{5} C_{\mathrm{OO}}=175.0000 \mathrm{eV} \AA,{ }^{5}$ and $e$ is the charge of an electron. The effective charges $q_{i}$ are $q_{\mathrm{O}}$ $=-1.2$ and $q_{\mathrm{Si}}=2.4 .^{25}$

As defined, the potential has the unphysical property of diverging to minus infinity at small distances. ${ }^{26}$ An activation barrier of approximately $7000 \mathrm{~K}$ is needed to get to these distances and therefore, for temperatures below this, the original potential causes no problems. Simulations that exceed this temperature will allow some of the ions to cross 


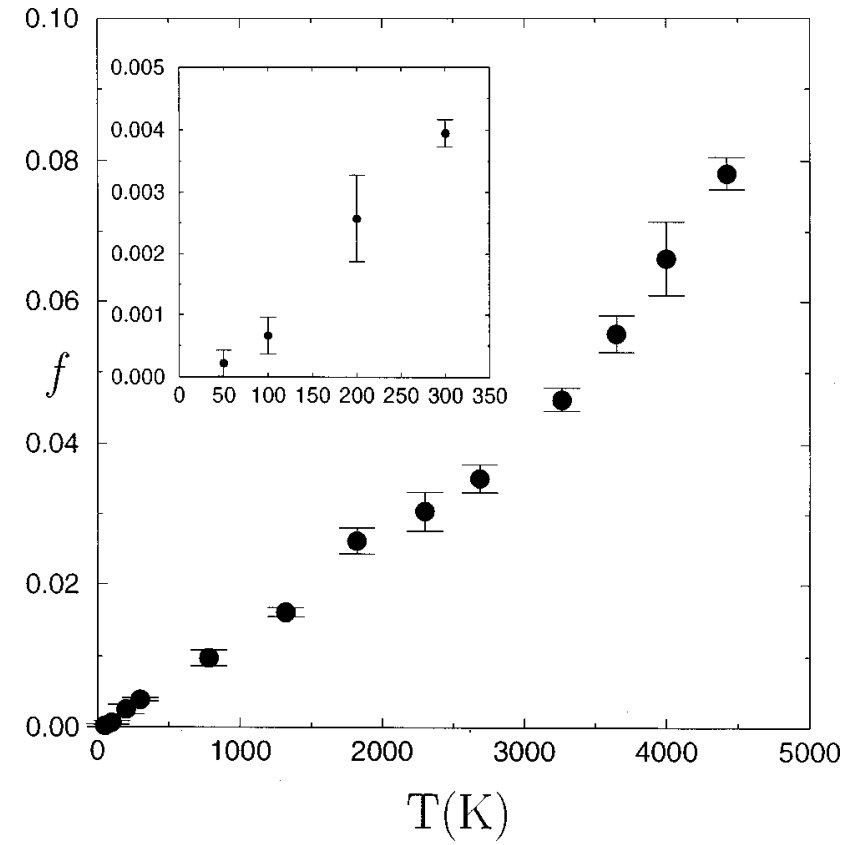

FIG. 2. The fraction of imaginary frequency modes as a function of temperature. For clarity, the values at very low temperature are also shown in the inset.

this barrier. To prevent this, the original potential is replaced with a harmonic potential when $r_{i j}$ is smaller than the location of the barrier, which occurs at $r_{i j} \leqslant 1.1936 \AA$ and $r_{i j}$ $\leqslant 1.439 \AA$ for $\mathrm{Si}-\mathrm{O}$ and $\mathrm{O}-\mathrm{O}$ interactions, respectively. ${ }^{26}$ The Coulomb part of the potential was calculated using the Ewald sum method ${ }^{27,28}$ with a convergence parameter, $\alpha$ $=0.301$ and by using all the wave vectors $\mathbf{k}$ with $|k|$ $\leqslant 6(2 \pi / L) ; L$ is the length of the simulation box. The shortranged interaction was truncated at a distance of $5.5 \AA$. In order to ensure that it vanished smoothly at the cutoff distance, the original potential was modified by the addition of $A r^{4}+B$, where the constants $A$ and $B$ were determined by requiring the potential and the force to vanish at the cutoff.

The system studied in this work consisted of $216 \mathrm{Si}$ and $432 \mathrm{O}$ atoms with masses of 28.0086 and $15.9994 \mathrm{amu}$, respectively, and periodic boundary conditions in all directions. The pressure was held constant at $P=0$ using an Andersen piston ${ }^{29}$ with a piston mass of $6 \times 10^{-3}$ amu $\AA^{-4}$ and velocity rescaling at every time step to maintain fixed temperature. The equations of motion were integrated, with a time step of $1.6 \mathrm{fs}$, using a modified velocity-Verlet algorithm, ${ }^{30-32}$ which eliminated the need for scaled coordinates. The system was initialized to a diamond lattice and heated to $7000 \mathrm{~K}$ after which it was equilibrated for 100000 time steps. This initial configuration was then cooled to $50 \mathrm{~K}$ at a constant rate of $1.77 \times 10^{13} \mathrm{~K} / \mathrm{s}$. At this cooling rate, the system will fall out of equilibrium at about $3000 \mathrm{~K}$, which is higher than the experimental glass transition for silica occurring around $1400 \mathrm{~K}$. Although this cooling rate is quite large, we do not expect that our qualitative conclusions will be greatly affected. (For a detailed discussion of cooling rate effects in simulations of silica using the BKS potential, see Ref. 26). During the course of the run, coordinates were saved at selected temperatures. These initial sets were used

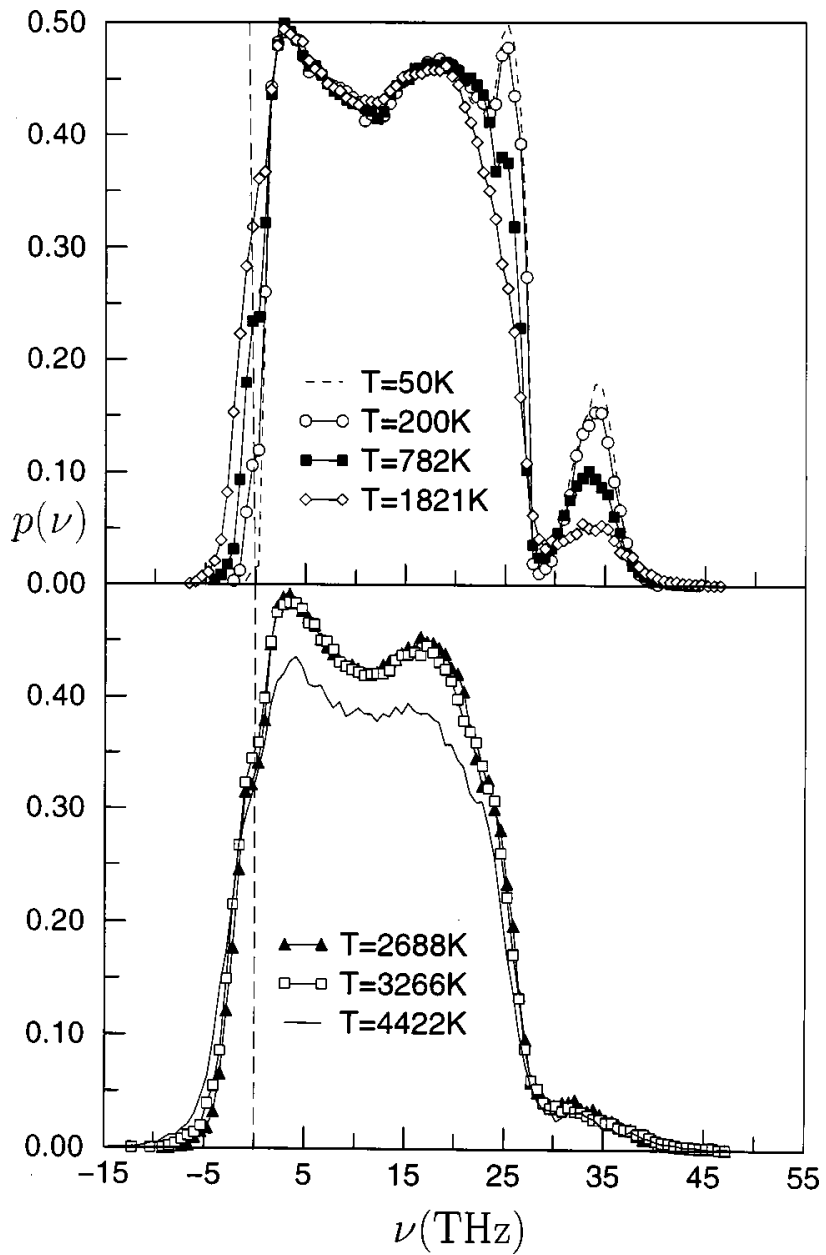

FIG. 3. The full participation ratios as a function of frequency at several temperatures for $\mathrm{SiO}_{2}$. For display purposes, the imaginary frequencies are plotted along the negative axis.

to generate six more sets, which were each relaxed for approximately 100000 steps. The results shown here are the average of these seven sets.

The calculated INM DOS, as a function of frequency, for our systems is shown in Fig. 1 for a variety of temperatures. For convenience, the imaginary modes are plotted along the negative frequency axis. The most obvious trend of these plots is as temperature increases the two sharp peaks at high frequency (roughly corresponding to transverse and longitudinal optical modes) ${ }^{10}$ disappear, eventually converging to a diffuse shoulder at temperatures above the glass transition. Also, the fraction of imaginary frequency modes, given by the area under the imaginary frequency region of the DOS and plotted in Fig. 2, increases, as expected, as the temperature is increased. Note that, the fraction of imaginary frequency modes is nonzero at all nonzero temperatures. This is a unique feature of amorphous solids, as opposed to crystalline solids, indicating the presence of anharmonic directions in the potential energy surface at all temperatures. Another feature seen in Fig. 2 is the sudden increase in slope of the fraction of imaginary modes at temperatures near the glass transition. Such a change in slope was also seen in the INM analysis of simple materials such as a Lennard-Jones glass and a system of particles interacting with a $r^{-6}$ potential, ${ }^{13}$ 


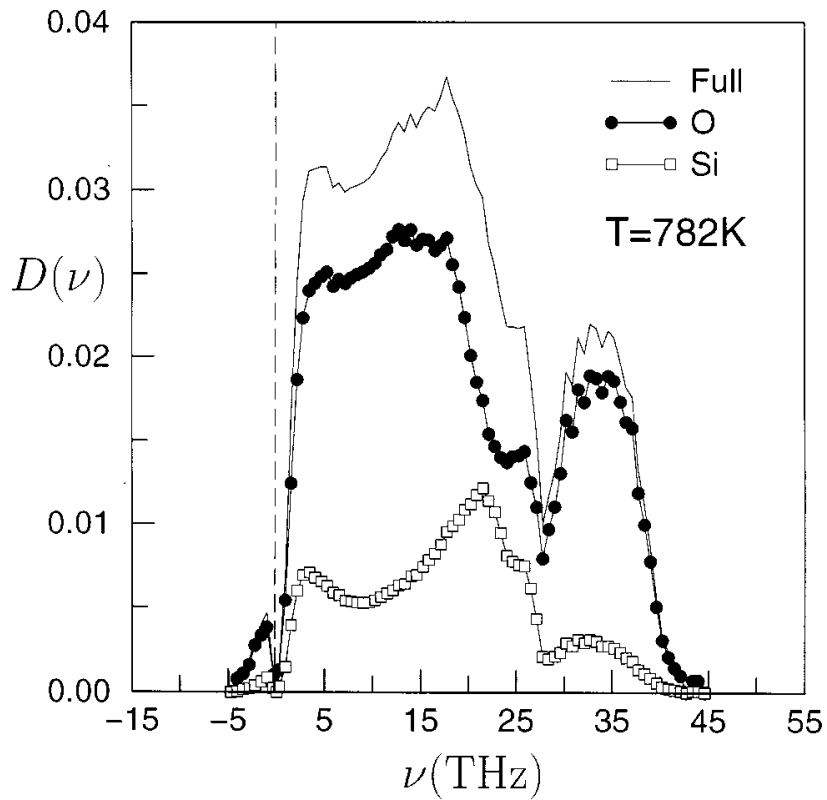

FIG. 4. The projected and full densities of states vs frequency for silica at a temperature of $782 \mathrm{~K}$.

where it was attributed to the onset of extended imaginary frequency modes.

To further understand the nature of the imaginary frequency modes we must examine the eigenvectors as well as the eigenfrequencies discussed in the previous paragraph. In Fig. 3 are shown the configurationally averaged participation ratios, $p(\nu)$, as functions of frequency at the temperatures studied. Consistent with what was seen in our earlier INM work on simple glasses and in the $T=0 \mathrm{~K}$ normal mode study of silica by Taraskin and Elliot, the high- and lowfrequency extremes of the DOS exhibit disorder-induced localization indicated by the small participation ratio values in those regions. (For silica, one also sees localization in the gap between the acoustic and optical bands.) An important consequence of this band edge localization is that the imaginary frequency modes are seen to have very low participation ratios at very low temperature, indicating that, at those temperatures, the highly anharmonic directions in the potential energy surface represent localized motions. As temperature increases up to and above the glass transition temperature the value of the participation ratio at the boundary between the real and imaginary regions of the DOS steadily increases until a saturation at about 0.33 is reached, indicating a fundamental change in the nature of the anharmonic directions of the potential energy surface from being primarily localized to having some extended character.

Since silica consists of two different types of atoms, $\mathrm{Si}$ and $\mathrm{O}$, it is possible to project the eigenvectors onto each of the two types and examine separately the projected DOS for $\mathrm{Si}$ and $\mathrm{O}$. These quantities for a system at a temperature of $782 \mathrm{~K}$ are shown together with the full DOS in Fig. 4. Ideally, if the atomic motions represented by a given eigenvector were independent of atom type, the projected DOS for oxygen should be twice that of silicon simply on the basis of stoichiometry; however, Fig. 4 indicates that the typical motions in the imaginary frequency regime (as well as in the

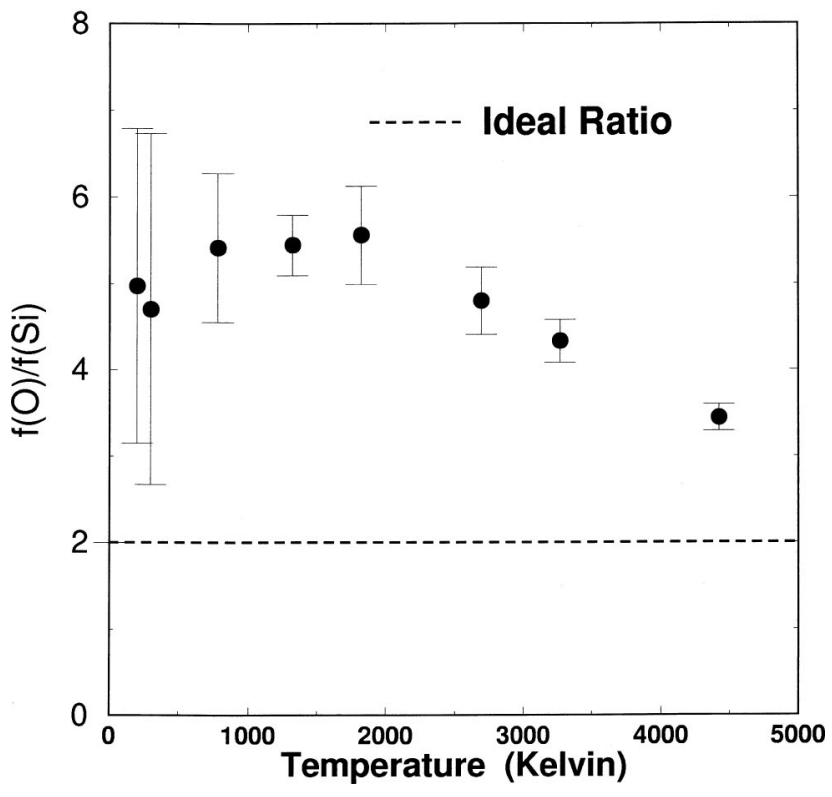

FIG. 5. The ratio of the fraction of imaginary frequency modes projected on to oxygen atoms to that ratio for the silicon atom projections as a function of temperature.

high frequency optical band) are oxygen dominated, even taking into account the stoichiometry difference. To examine this further, we plot, in Fig. 5 the ratio of the fraction of imaginary frequency modes projected onto oxygen to the same quantity for silicon. As mentioned before, ideally, this should have a value of 2.0 if the eigenvector has no bias for a particular type of atom. Instead, for temperatures below $T_{g}$ the ratio is nearly constant at a value just above 5 and begins to fall off linearly above $2000 \mathrm{~K}$, indicating that the highly anharmonic motions responsible for relaxation at temperatures below the glass transition are primarily localized motions of oxygen atoms. This is consistent with previous neutron scattering results showing the presence of nonacoustic localized modes at low temperature in silica, which have been interpreted as coupled rotations of a few $\mathrm{SiO}_{4}$ tetrahedra. ${ }^{33}$ Such motions are dominated by oxygen motion, since the tetrahedra rotate about the $\mathrm{Si}$ centroid, which remains approximately fixed. To show this explicitly, we examine directly the eigenvectors for two randomly selected imaginary frequency modes from different samples at $100 \mathrm{~K}$. Figure 6 show ball and stick images of the ten Si (gray) and $\mathrm{O}$ (black) atoms with the largest eigenvector projections in each modes. Also plotted are all atoms bonded to these 10 (shown in white). (In both of these configurations, the 10 atoms represent about $60 \%$ of the eigenvector length.) The arrows represent the eigenvector projection onto each atom. From this, it can be clearly seen that the dominant contribution to each eigenvector are coupled rotations of $\mathrm{SiO}_{4}$ units. In addition, for these modes the coupled tetrahedra do not form a compact cluster, but instead form chains-this structure was typical of all localized modes so examined.

\section{SUMMARY}

We have examined the dynamics and potential energy surface of amorphous and supercooled silica using instanta- 


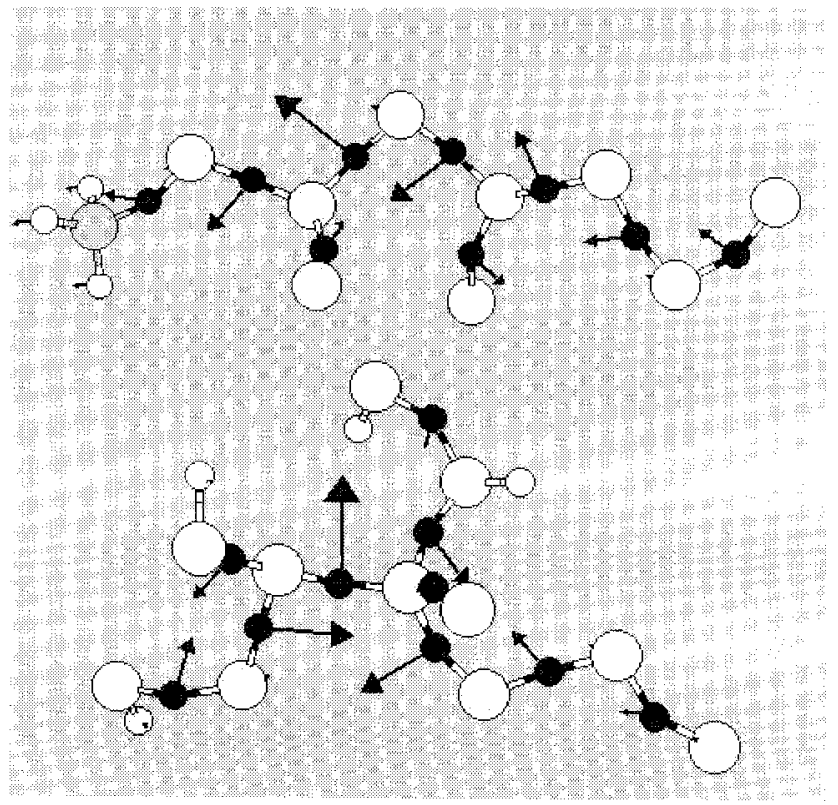

FIG. 6. Ball and stick representations of two representative imaginary frequency localized eigenvectors taken from two separate simulation samples at $100 \mathrm{~K}$. The ten atoms with the largest eigenvector projection are shown in black (oxygen) and gray (silicon). Also shown are all atoms (shown in white) of the system attached to these top 10 . The projected eigenvectors on each atom are shown as arrows. The frequencies and participation ratios (shown in parentheses) for the top and bottom configurations are $i \nu$ $=-0.91 \mathrm{THz}[p(\nu)=0.028]$ and $i \nu=-1.76 \mathrm{THz}[p(\nu)=0.021]$, respectively.

neous normal mode analysis of configurations generated by molecular dynamics simulation at a variety of temperatures. The interatomic interactions are modeled using the silica potential of BKS. ${ }^{25}$ At low temperatures, below the glass transition, we find that the imaginary frequency modes, which represent the highly anharmonic collective motions in the glass, are localized, coupled rotations of $\mathrm{SiO}_{4}$ tetrahedral units, consistent with neutron scattering experiments ${ }^{5}$ and earlier normal mode analysis of $T=0 \mathrm{~K}$ simulation data. ${ }^{10} \mathrm{In}$ addition, we observe that the coupled tetrahedra are grouped in chains as opposed to compact clusters. As the temperature is increased we observe a fundamental change in the nature of the highly anharmonic region of the potential energy surface at about $2500 \mathrm{~K}$, as evidenced by a sudden increase in the slope of the fraction of imaginary frequency modes as a function of temperature and a rapid increase in the amount of silicon participation in the imaginary modes, which at low temperatures is small due to the dominance of $\mathrm{SiO}_{4}$ tetrahedral units (which leaves the Si more or less fixed). We interpret this change in the nature of the region of potential energy surface sampled by the system to be due to the ap- pearance of a new class of extended imaginary frequency modes at the higher temperatures. Although any direct relationship is at present speculative, this behavior is similar to that reported in recent simulation studies of supercooled liquid silica using the BKS model ${ }^{34}$ which observed abrupt changes in the temperature dependences of the diffusion constant and viscosity at $3300 \mathrm{~K}$, which they interpret as indicating a change in the mechanism of transport from hopping to continuous flow.

\section{ACKNOWLEDGMENTS}

The authors gratefully acknowledge support from the Petroleum Research Fund under Grant No. 30970-AC5 and from the Kansas Center for Advanced Scientific Computing for the use of their computational resources.

${ }^{1}$ P. W. Anderson, B. I. Halperin, and C. M. Varma, Philos. Mag. 25, 1 (1972).

${ }^{2}$ W. A. Phillips, J. Low Temp. Phys. 7, 351 (1972).

${ }^{3}$ R. O. Pohl and E. T. Swartz, J. Non-Cryst. Solids 76, 117 (1985).

${ }^{4}$ U. Buchenau, M. Prager, N. Nucker, A. J. Dianoux, N. Ahamad, and W. A. Phillips, Phys. Rev. B 34, 5665 (1986).

${ }^{5}$ U. Buchenau, M. Prager, W. A. Kamitakahara, H. R. Shanks, and N. Nucker, Europhys. Lett. 6, 695 (1988).

${ }^{6}$ U. Buchenau, C. Pecharroman, R. Zorn, and B. Frick, Phys. Rev. Lett. 77, 659 (1996).

${ }^{7}$ B. B. Laird and H. R. Schober, Phys. Rev. Lett. 66, 636 (1991).

${ }^{8}$ L. D. van Ee, B. J. Thijsse, and J. Sietsma, Phys. Rev. B 57, 906 (1998).

${ }^{9}$ A. Angell, Science 267, 1924 (1995).

${ }^{10}$ S. N. Taraskin and S. R. Elliot, Europhys. Lett. 39, 37 (1997).

${ }^{11}$ S. N. Taraskin and S. R. Elliot, Phys. Rev. B 59, 8572 (1999).

${ }^{12}$ S. D. Bembenek and B. B. Laird, Phys. Rev. Lett. 74, 936 (1995).

${ }^{13}$ S. D. Bembenek and B. B. Laird, J. Chem. Phys. 104, 1 (1996).

${ }^{14}$ R. M. J. Cotterill and J. U. Madsen, Phys. Rev. B 33, 262 (1986).

${ }^{15}$ B. Madan, T. Keyes, and G. Seeley, J. Chem. Phys. 92, 7565 (1990).

${ }^{16}$ T. Keyes, J. Chem. Phys. 101, 5081 (1994).

${ }^{17}$ M. Cho, G. R. Fleming, S. Saito, I. Ohmine, and R. Stratt, J. Chem. Phys. 100, 6672 (1994).

${ }^{18}$ R. Stratt, Acc. Chem. Res. 28, 201 (1995).

${ }^{19}$ T. Keyes, J. Phys. Chem. A 101, 2921 (1997).

${ }^{20}$ M. C. C. Ribiero and P. A. Madden, J. Chem. Phys. 108, 3256 (1998).

${ }^{21}$ F. Sciortino and R. Tartaglia, Phys. Rev. Lett. 78, 2385 (1997).

${ }^{22}$ E. La Nave, A. Scala, F. W. Starr, F. Sciortino, and H. E. Stanley, Phys. Rev. Lett. 84, 4605 (2000).

${ }^{23}$ J. D. Gezelter, E. Rabani, and B. J. Berne, J. Chem. Phys. 107, 4618 (1997).

${ }^{24}$ R. J. Bell and P. Dean, Discuss. Faraday Soc. 50, 55 (1970).

${ }^{25}$ B. W. H. van Beest, G. J. Kramer, and R. A. van Santen, Phys. Rev. Lett. 64, 1955 (1990).

${ }^{26}$ K. Vollmayr, W. Kob, and K. Binder, Phys. Rev. B 54, 15808 (1996).

${ }^{27}$ P. Ewald, Ann. Phys. (Leipzig) 64, 253 (1921).

${ }^{28}$ L. V. Woodcock and K. Singer, Trans. Faraday Soc. 67, 12 (1971).

${ }^{29}$ H. C. Andersen, J. Chem. Phys. 72, 2384 (1980).

${ }^{30}$ D. Brown and J. H. R. Clarke, Mol. Phys. 51, 1243 (1984).

${ }^{31}$ M. A. Allen and D. J. Tildesley, Computer Simulation of Liquids (Oxford Science, Oxford, 1987).

${ }^{32}$ S. D. Bembenek, Thesis dissertation, University of Kansas, August 1997.

${ }^{33}$ U. Buchenau, H. M. Zhou, N. Nucker, K. S. Gilroy, and W. A. Phillips, Phys. Rev. Lett. 60, 1318 (1988).

${ }^{34}$ J. Horbach, W. Kob, and K. Binder, Phys. Rev. B 60, 3169 (1999). 